\title{
Second-order isomorphism of internal representations of familiar faces
}

\author{
IAN E. GORDON and SANDRA HAYWARD \\ Lnilersity of Exeter, Exeter, EX4 $4 Q G$, Devon, England
}

\begin{abstract}
Following a suggestion by Shepard and Chipman (1970), photographs and names of 15 well-known faces were used as stimuli in judgments of similarity. Ss ranked all pairs of stimuli in terms of facial similarity either from memory when presented with pairs of names, or from photographs. There was a highly significant positive correlation between these two types of judgment. A simple classificatory analysis of the 15 faces in terms of the physical features provided evidence that judgments based on the internal representation of the faces utilized pictorial information. These results extend the generality of Shepard and Chipman's findings and confirm the usefulness of an approach to the problem of mental images based on the concept of second-order isomorphism.
\end{abstract}

Shepard and Chipman have pointed out that questions sometimes raised about the nature of internal representations or mental images apparently presuppose a discredited first-order structural isomorphism between stimuli and their representations. The investigation of internal representations or images can proceed, however, if we adopt a strategy based upon the concept of second-order isomorphism (Shepard, 1968). What should be studied is not the relation between object and internal representation, but rather the second-order correspondence linking the relations between different external stimuli and the relations between their internal representations.

Shepard and Chipman's Ss ranked all possible pairs of outline drawings of 15 American states for degree of similarity (the "picture" condition). Prior to this, the Ss performed the same ranking from memory when presented with pairs of names of the states (the "name" condition). The correlation between the group average rankings of pairs in the two conditions was positive and highly significant. Individual scores were subjected to a multidimensional scaling analysis which showed, among other things, that it was highly probable that under both picture and name conditions it was mainly the geometric or pictorial properties of the actual shapes which formed the basis of Ss' judgments.

The study reported here arose out of an explicit suggestion by Shepard and Chipman that their procedure might be repeated, using familiar faces. We present results that confirm the usefulness of the approach to the problem via second-order isomorphism. In addition, a simple classificatory analysis of the facial stimuli is described which suggests that in our name condition, where judgments are based upon internal representations only, pictorial properties of the original stimuli influence Ss' judgments.

\section{METHOD}

Judgments of similarity were obtained, using pairs of stimuli which were either (a) photographs of well-known people or (b) their names.
Stimuli

\section{The Picture Condition}

Photographs of 15 faces well known to British Ss were used in the picture condition. Three categories of famous people were used: (a) TV and film personalities-Benny Hill, Michael Aspel, Harry Corbett, Ringo Starr, David Frost; (b) British politicians-Wilson, Heath, Brown, Douglas-Home, Churchill; (c) foreign statesmen-Nixon, de Gaulle, Kosygin, Mao, Trudeau.

The faces were recopied from posed photographs appearing in newspapers and magazines. They were printed so as to be approximately the same size. The faces were seen either in full view or slightly to one side. Pairs of photographs, each $7.5 \mathrm{x}$ $6.5 \mathrm{~cm}$, were mounted side by side on $9 \times 14 \mathrm{~cm}$ white cards. All possible (105) pairs of photographs were prepared, each face appearing equally of ten on the left and right.

\section{The Name Condition}

Cards in the name condition were arranged exactly as in the picture condition, except that pairs of names, printed in uppercase letters, replaced the faces.

\section{Procedure}

Single pictures were presented in a preliminary screening procedure. Only those Ss who could quickly and easily name all 15 faces were used in the experiment. Ss were tested on two occasions separated by at least $48 \mathrm{~h}$. On the first occasion, the 105 name cards were presented with instructions to rank order, in terms of degree of similarity, the pairs of faces associated with the names. On the second occasion, the ranking procedure was repeated using pairs of pictures. Times to complete the ranking ranged from $45 \mathrm{~min}$ to $1 \mathrm{~h} 30 \mathrm{~min}$, in the name condition, and from $30 \mathrm{~min}$ to $1 \mathrm{~h} 10 \mathrm{~min}$, in the picture condition.

\section{Subjects}

The eight Ss, four male and four female, were students and staff at Exeter University.

\section{RESULTS AND DISCUSSION}

Rank order correlation coefficients (Spearman's rho) were derived from the two sets of ranks obtained from each $\mathrm{S}$. Then the average rank value assigned to each name and picture card was used in the calculation of the overall correlation between name and picture data. 
Table 1

Facial Attributes of the 15 Photographs Used in the Picture Condition

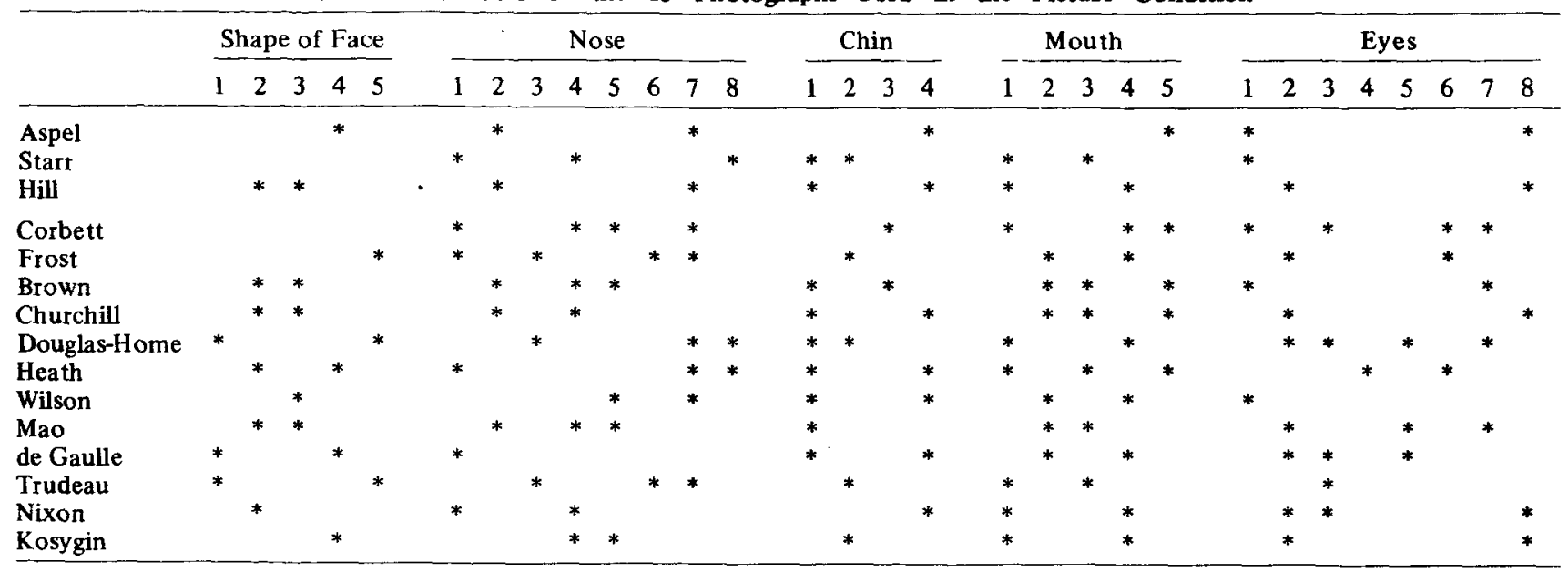

Note-* indicates agreed presence of a feature

Finally, the consistency of judgments was assessed, using a coefficient of concordance, Kendall's W (Siegel, 1956), with the eight sets of name ranks and then the eight sets of picture ranks. The results of these statistical analyses are summarized below.

\section{Individual Rankings}

All rank correlations were positive. The rho values for individual Ss ranged from .39 to .73 , with a mean (and a median) value of .54 . All correlations were highly significant $(p<.001)$.

\section{Group Name vs Group Picture Rankings}

The overall rank order correlation between the name and picture conditions was +.93 . This result is highly significant $(\mathrm{p}<.001)$.

\section{Concordance Coefficients}

For the eight sets of picture rankings, the calculated value for $W$ was $.33(\mathrm{p}<.001)$. Not surprisingly, the value of $W$ in the name condition was lower at .17 $(p<.01)$, a result which indicates that if the Ss were using visual images in the name condition, these were not as good as perceptual images resulting from the actual photographs. Although statistically significant, the $\mathrm{W}$ values are low, a result which reflects the extreme difficulty involved in rank ordering 105 pairs of stimuli.

From the preceding results, we conclude that Ss are able to make similar judgments using either actual stimuli or their internal representations, and this confirms Shepard and Chipman's original finding.

It is now necessary, as Shepard and Chipman recognized, to try to establish whether the correlations obtained are the result of Ss using pictorial cues in both experimental conditions. Shepard and Chipman showed that pictorial aspects of their outline shapes were the probable basis of Ss' judgments by subjecting their data to a multidimensional scaling analysis. The material used in the present study is both more complex and more familiar than outline drawings of states. To pursue the question of the basis of Ss' judgments in the name condition, the faces used were subjected to a simple classificatory analysis.

\section{Analysis of Faces}

Examination of the 15 photographs used in the experiment suggested 30 different physical features that could be used to differentiate between them. The features were grouped as follows: (a) shape of face-thin, fat, round, oval, triangular; (b) nose-long, short, thin, fat, rounded, pointed, straight, hooked; (c) chin-indistinct, pointed, square, rounded; (d) mouth-wide, narrow, both lips full, both lips thin, top lip thin; (e) eyes-large, small, deep set, not deep set, close set, wide apart, eyebrows prominent, eyebrows indistinct. This ordering of features is maintained in Table 1.

Ten new Ss, students and staff from the Exeter department, rated each photograph for the presence or absence of the features listed above. Note that (a) the features are not all mutually exclusive: a face could be rated thin and triangular; (b) the analysis is crude, in that it would not be possible to guarantee recognition of any of the faces from a verbal description based only upon a selection of the $\mathbf{3 0}$ features; (c) only simple visual aspects of faces were dealt with: more subtle factors such as apparent age were not included in the analysis; (d) Ss were asked to rate quickly and without too much deliberation; they were not given precise verbal definitions of the meanings of "close set," "thin," etc. Table 1 shows the commonly agreed classifications of the 15 faces. (Each asterisk indicates that a majority 
of judges agreed as to a feature s presence.)

The 10 pairs of faces judged most alike and the 10 pairs judged least alike in the name condition were then examined to see how many of the 30 features the pairs were judged as having in common. The first 10 pairs of faces in the name condition, in the order 1.10 , were: Aspel-Trudeau, Brown-Mao, Frost-Heath, Brown-Churchill, Wilson-Hill, Wilson-Brown, Heath-Nixon, Churchill-Mao, Starr-Corbett, Home-Frost. The 10 pairs of faces ranked least alike in the name condition, starting at Rank 105, were: Mao-Home, Frost-Churchill, Mao-Aspel, Mao-Frost, Home-Brown, Kosygin-Mao, Corbett-Mao, Home-Wilson, Hill-Home, Mao-Starr. Reference to Table 1 will confirm that the first 10 pairs of faces had a total of 54 features shared by members of pairs. The 10 pairs judged least alike had a total of only 27 features in common. These totals were compared using the binomial test (Siegel, 1956). The difference between them is highly significant $(Z=2.89$, $\mathrm{p}<.005$ ).

It is highly probable that in the name condition, judgments were not based only upon simple visual aspects of faces. For example, questioning of Ss revealed that the Aspel-Trudeau pair appeared similar partly because of the similarity in apparent age and also because both faces were judged as handsome. This helps explain the otherwise paradoxical result that this pair of faces was judged most similar of all in the name condition but shared only a single feature in the classificatory analysis.

It appears, then, that knowing something of the physical appearance of stimuli is a good basis for predicting the outcome of judgments made using their representations, and that the judgments involve the use of some information of a pictorial nature. Ss' reports on completion of the name condition made it clear that in their opinion, they had been using pictorial properties in their judgments. In particular, most Ss stated that it was the remembered shapes and sizes of noses which mostly influenced their judgments, followed by overall shapes of faces; other aspects of the faces were used to a lesser extent. Further examination of the classification in Table 1 shows that 29 of the 54 features shared between the 10 pairs ranked most alike relate to the nose and shape of the face. The 10 pairs of faces ranked least alike have no shape of the face features in common and have a total of only 10 nose features in common.

We interpret these results as confirming the visual or pictorial contribution to judgments in the name condition, even though the analysis has been a crude one. But, as we have previously stated, this confirmation does not exclude the possibility that other factors, such as age and status of the person portrayed and even Ss' likes and dislikes, may influence judgment in both name and picture conditions. It may, however, be significant that an analysis very different in kind from that performed by Shepard and Chipman should have led to the same conclusion concerning the basis of judgments in the name condition.

\section{Imaging}

We wish to report an unusual subjective effect which was mentioned spontaneously by all Ss and which we also experienced for the first time while ranking in the name condition. Rank ordering two pairs of faces for similarity using only internal representations is an unusual task. To do this at least 105 times, with each representation having to be produced many times in all, induces marked feelings of strain. After $45 \mathrm{~min}$ or so, there is a distinct impression of emptiness, of having temporarily exhausted the ability to retrieve and "see" the stored information. It seems to us quite possible that with nonverbal stored material, intensive, prolonged, and paced retrieval for comparison or manipulation of representations might eventually lead to blocks, periods when the use of the internal representations becomes temporarily impossible.

\section{CONCLUSIONS}

As has been acknowledged, the present study originated in a suggestion by Shepard and Chipman (1970). The results increase the generality of their findings and confirm the usefulness of their approach to the problem of imagery via second-order isomorphism. It is possible that this approach may be developed still further, for it circumvents an embarassing problem facing those who have studied imagery-the person who denies ever having had an image. Logically, if such a person can recognize visual stimuli, he must have internal representations of them. The present approach facilitates the investigation of these representations, including, for example, the extent to which they are pictorial, without any necessary concern over the problem of subjective awareness of images. Making Ss manipulate images may be more fruitful than recording their descriptions.

\section{REFERENCES}

Shepard, R. N. Cognitive psychology: A review of the book by U. Neisser. American Journal of Psychology, 1968, 81, 285-289.

Shepard, R. N., \& Chipman, S. Second-order isomorphism of internal representations: Shapes of states. Cognitive Psychology, 1970, 1, 1-17.

Siegel, S. Nonparametric statistics. New York: McGraw-Hill, 1956.

(Received for publication March 12, 1973; revision received May 9, 1973.) 